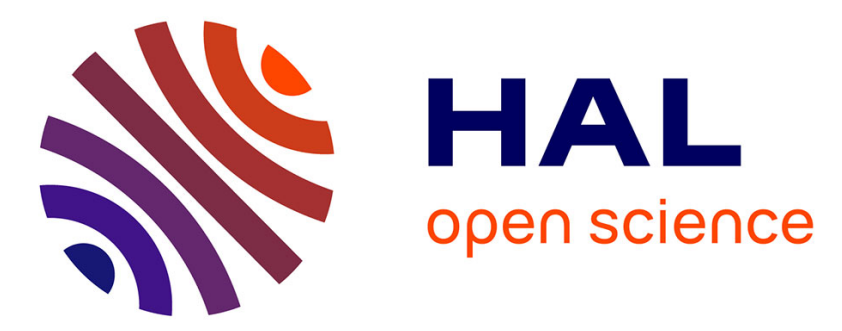

\title{
Trinary mappings: a tool for the determination of potential spectral paths for optical monitoring of optical interference filters
}

Mael Vignaux, Fabien Lemarchand, Thomas Begou, Catherine Grezes-Besset, Julien Lumeau

\section{To cite this version:}

Mael Vignaux, Fabien Lemarchand, Thomas Begou, Catherine Grezes-Besset, Julien Lumeau. Trinary mappings: a tool for the determination of potential spectral paths for optical monitoring of optical interference filters. Applied optics, 2018, 57 (24), 10.1364/AO.57.007012 . hal-01870540

\author{
HAL Id: hal-01870540 \\ https://hal.science/hal-01870540
}

Submitted on 13 Mar 2019

HAL is a multi-disciplinary open access archive for the deposit and dissemination of scientific research documents, whether they are published or not. The documents may come from teaching and research institutions in France or abroad, or from public or private research centers.
L'archive ouverte pluridisciplinaire $\mathbf{H A L}$, est destinée au dépôt et à la diffusion de documents scientifiques de niveau recherche, publiés ou non, émanant des établissements d'enseignement et de recherche français ou étrangers, des laboratoires publics ou privés. 


\title{
Trinary mappings: a tool for the determination of potential spectral paths for optical monitoring of optical interference filters
}

\author{
Mael Vignaux, ${ }^{1}$ Fabien Lemarchand,${ }^{1}$ Thomas Begou, ${ }^{1}$ Catherine Grezes- \\ BESSET, ${ }^{2}$ JuLIEN LUMEAU ${ }^{1, *}$ \\ ${ }^{1}$ Aix-Marseille Univ, CNRS, Centrale Marseille, Institut Fresnel, Marseille, France \\ ${ }^{2}$ CILAS, Etablissement de Marseille, Pole ALPHA Sud - Saint Mitre, 13400 Aubagne, France \\ *Corresponding author: julien.lumeau@fresnel.fr
}

Received XX Month XXXX; revised XX Month, XXXX; accepted XX Month XXXX; posted XX Month XXXX (Doc. ID XXXXX); published XX Month XXXX

An alternative new technique for the determination of efficient optical monitoring strategy of optical interference filters is presented. This technique relies on the analysis of optical monitoring signals for different optical monitoring wavelengths and the comparison of these signals with some pre-defined criteria in order to generate potential spectral path compatible with a dedicated trigger point monitoring technique. Trinary mappings are then generated in order to determine possible optical monitoring strategies. This technique is finally implemented on various filter designs and experimentally validated.

OCIS codes: (310.0310) Thin films; (310.4165) Multilayer design; (310.1620) Interference coatings.

http://dx.doi.org/10.1364/AO.99.099999

\section{Introduction}

Optical interference filters offer a very broad range of optical functions for the control of the spectral properties of light. With the last 15-year improvement of both the design techniques and the manufacturing systems, it is now very common to fabricate very complex filters with very large number of layers and totally aperiodic structures [1]. In order to secure low errors on the thickness of each of the layers of the stack, it is best to combine stable deposition systems as provided by sputtering technique and in-situ optical monitoring [2,3]. By combining different algorithms based on optical criteria such as turning point monitoring (determination of the moment the derivative of the optical monitoring signals cancels) or trigger point monitoring (determination of pre-defined optical monitoring signal levels) it is possible to accurately stop the deposition of each of the layers [4]. More advanced versions of trigger point monitoring can be used, they are known Percentage Of Extremum Monitoring (POEM) [4] or optical monitoring by swing [5] and consist in taking into account the amplitudes of the previous extrema of the optical monitoring signal to correct for the trigger point monitoring value. However, while such techniques can accurately determine the moment a deposition has to be stopped, the accuracy highly depends on the selected optical monitoring wavelength. The choice of the optimal optical monitoring wavelength is therefore a critical step that will directly affect the final performances of the filter. Indeed, with some classical $[4,6]$ or very complex filters [7], the error can quickly diverge and result in large discrepancies if the optical monitoring wavelengths are not properly selected.

While it is obvious that this critical problem has drawn large interest in the optical thin-film community, there have been quite a small amount of works published. Indeed, it is obvious that there have been a wide range of progresses in the domain. This is clearly illustrated by the increased complexity and the lower discrepancy of the fabricated filters [8]. However, most of the efforts have been carried out by industrials resulting in a limited spreading of the results and limited scientific work on the topic. We are aware of only a few academic studies on the topic. Several papers have been published by R. Willey, but mostly on some very specific filters and without generalization of the methods to filters with arbitrary structures [4,8-10]. A. Zoeller has also widely contributed to the domain, in particular in the improvement of optical monitoring systems [11] or virtual deposition process [10-12]. Finally, the main general investigations have been carried out by A. Tikhonravov and M. Trubetskov. For example, in ref. [13] they provide a full investigation on how to find the best possible monitoring wavelengths in the case of a full Level Cut monitoring. For a given multilayer stack, they provide a monitoring spreadsheet that contains the sequence of monitoring wavelengths and their corresponding termination level. For the creation of such a spreadsheet they propose to follow different steps in order to perform a constrained optimization with several limited criteria:

- The number of different monitoring wavelengths is limited up to 5 . Hence, depending on the spectral region attainable with a given monitoring system and the maximum allowed number of optical 
monitoring wavelengths an optimal monitoring spreadsheet can be created.

- The amplitude (A) of the signal between its maximum $V_{\max }$ and its minimum $V_{\min }$ has to be defined by the user. In the case that signal extrema are not achieved during layer deposition (for example, a layer is too thin), they consider a virtual increase of its thickness until both extrema are finally located in order to calculate the final amplitude.

- The initial and final signal $\boldsymbol{S w i n g}$ (Sin and $\mathrm{S}_{\text {fin }}$ ) value are also user defined. It reflects the ratio between the amplitude and the distance separating the last turning point from the trigger point of the layer $\left(\mathrm{S}_{\text {fin }}\right)$ but also between the amplitude and the distance separating the initial value of the signal from the first extrema ( $\left.\mathrm{S}_{\mathrm{ini}}\right)$.

- The distance $\Delta$ from the Trigger Point to the next Turning Point should also be defined. For some monitoring devices this is quite important value and should not be too small.

The strategy elaborated by the computer can then be considered as resulting from an optimization problem, with strong boundaries for the calculation algorithm. Indeed, a monitoring function $\mathrm{Fm}_{\mathrm{m}}$ which is used as a merit function needs to be minimized. This function uses weighing factors for each of these criteria that are predefined. This algorithm then allows one to find in a matter of minutes a possible strategy (and spreadsheet) for the desired stack within set boundaries. This is this type of strategy which is implemented in commercial software such as Optilayer [15].

However, one can see that this technique uses weights in the merit function, and it is hard to precisely determine the most efficient values for the parameters as it is case dependent. Also, the technique provides a single path that might include some layers that are not compatible with optical monitoring and would require time or quartz-based monitoring.

In order to propose an alternative method for the determination of the efficient optical monitoring strategy, we recently proposed an alternative approach based on the determination of the optical monitoring wavelength that allows minimizing the root mean square deviation of the reflected phase at a single wavelength. This technique consists in running virtual deposition process [10-12] and calculating the reflected phase at a single wavelength for each of the runs. Then, the optimal optical monitoring wavelength is the one that provides the lowest phase error at this wavelength. This technique was implemented on bandpass filters and was shown to be very efficient for the determination of all-optical monitoring strategies of this type of filters. However, this technique tends to be time-consuming as it relies on virtual deposition process simulation of deposition runs.

To overcome these limitations in term of calculation time for phasebased method and clear the tricky question of the determination of the weight parameters values in case of the method proposed by Trubetskov et al., we propose in this paper an alternative approach for the determination of possible optical monitoring strategies. It relies on a predetermined list of parameters as proposed by Trubetskov, but this list is slightly modified and completed with additional parameters. In addition, it does not intend to provide a single optical monitoring strategy but to help users to determine an efficient optical monitoring strategy, if not the best.

\section{Description of the method}

The principle of the algorithm that we have developed is first based on the generation of the optical monitoring signal for each of the wavelengths within a spectral region where optical monitoring can be implemented. Then, we analyze what are the critical parameters that need to be taken into account in order to minimize the layers thickness errors with trigger point monitoring strategy [4,5]. Finally, we combine these different criteria in order to define regions with high probability of success and use them to generate optimal paths for the creation of optical monitoring strategies based on phase calculation. There are some factors that will limit the optical monitoring accuracy of the thickness of each layers. Below is a non-exhaustive list:

- the distance to extrema of the signal that can vary from layer to layer,

- the final derivative of the signal when deposition needs to be triggered,

- $\quad$ the spectral resolution of the optical monitoring system as compared to the spectral fluctuations of the transmittance signal,

- the dynamic on every single layer,

- the transmittance value at trigger point,

- the number of extrema in the signal of a layer.

Let us analyze each of these criteria and define some criteria defining whether trigger point monitoring can be implemented safely or not.

\section{A. Criterion 1: Distance of the trigger point to extrema}

The distance a trigger point is located from the previous and the next extremum of the transmitted intensity modulation is a critical parameter that strongly influences the accuracy of the layer thickness monitoring. Indeed, trigger point monitoring is highly relying on the calculation of a ratio of distances between the trigger point and the last extremum as well as the distance between the last two extrema of the signal. Therefore, one can easily understand, that if trigger point monitoring is defined close to an extremum, in this case, the triggering criterion will occur in a region with low modulation of the signal that will be more sensitive to noise and that can be more affected by previous layer thickness errors. Actually, layer thickness error is minimum when trigger point is equidistant to the extrema of the intensity modulation and severely increases as soon as trigger point approaches an extremum. It can also be shown that thickness error is almost constant over broad spectral ranges when the distance to extrema is between 5 and $95 \%$ of the layer. This observation allows us to establish a first criterion that will secure low phase error even without any virtual deposition process runs, i.e. the trigger point intensity must be at a minimal distance of 5 and $95 \%$ before and after each extremum. This criterion is a necessary condition for minimizing phase errors but it is not a sufficient one as other parameters will affect the precision of the layer optical monitoring. We illustrated this criterion in Figure 1, in case of a single $\mathrm{Nb}_{2} \mathrm{O}_{5}$ layer.

Using this criterion it is possible to generate two different regions:

- $\quad$ trigger point monitoring -compatible regions (in blue) where the distance to the closest extremum of the signal is more than $2 \%$

- forbidden regions (in grey) where the distance to the closest extremum of the signal is less than $2 \%$

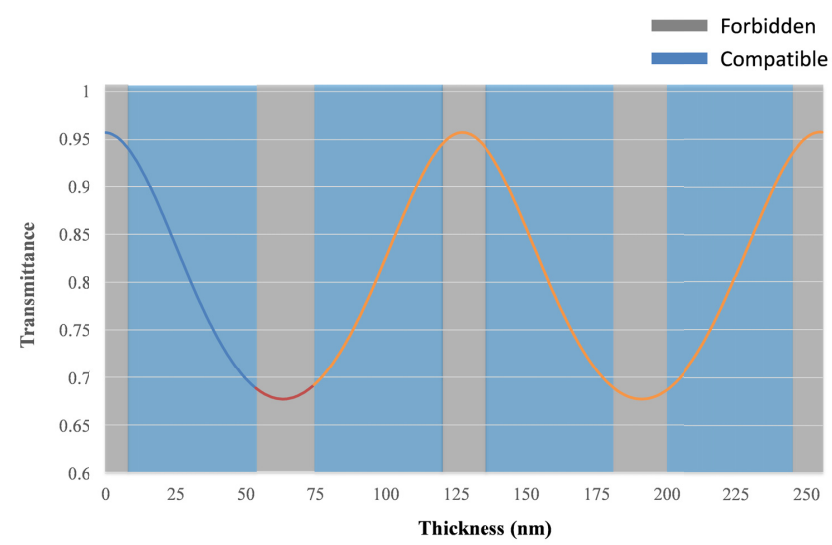


Figure 1. Illustration of the distance of the trigger point to extrema criterion.

\section{B. Criterion 2: Signal derivative at trigger point}

Another parameter to take into account when analyzing the efficiency of a trigger point monitoring-based optical monitoring strategy is the final derivative of the signal when triggering criterion is applied. This criterion is somewhat depending on the previous criterion as the signal slope will depend on the distance to the previous and next extremum. Indeed, regions where TPM technique would be applied, i.e. regions where zero derivative is reached at the end of the layer, is the worst condition for trigger point monitoring to be applied as it will induce very large errors. Conversely, if the slope is very large, it is clear that the distance between two measured intensities will be much larger than the noise level and high precision triggering point will be achieved, resulting in very small error on the final layer thickness (this analysis neglects any delay that would be associated with the signal processing if slope is very large). However, such a criterion also takes into account the amplitude of the modulation of the signal and therefore provides additional constraints. Actually, the differences in transmittance can be very large during the deposition of a multilayer structure. For example, it is the case when monitoring a quarter-wave dielectric mirror within the reflectance lobe where amplitude can vary very fast from one layer to another even if triggering point stays far from an extremum.

To define a criterion on the derivative, we first considered the noise of the optical monitoring signal. It can be defined using two values: the multiplicative and additive noise. By comparing this level of noise with the derivative of the signal, one can define the following criterion: the local derivative recalculated over a standard quartz thickness error window must be larger than the noise deviation. In other words, a wavelength will not be adapted for trigger point monitoring if the error associated with the optical monitoring of this layer at this specific wavelength is larger than the one associated with a quartz monitoring system (forbidden regions). We illustrated this criterion in Figure 2, in case of a single $\mathrm{Nb}_{2} \mathrm{O}_{5}$ layer. The criterion than we used is that derivative must be larger than \pm 0.0005 .

Using this criterion it is possible to generate two different regions:

- $\quad$ trigger point monitoring -compatible regions (in blue) where the recalculated amplitude based from the local derivative of the signal when deposition stop is triggered is larger than the noise standard level of the signal.

- forbidden regions (in grey) where the recalculated amplitude based from the local derivative of the signal when deposition stop is triggered is smaller than the noise standard level of the signal.

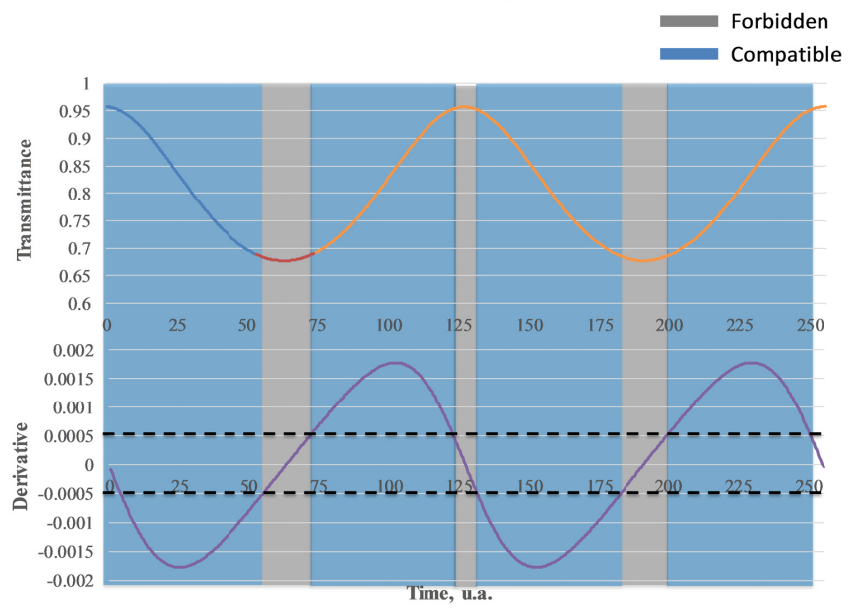

Figure 2. Illustration of the signal derivative at trigger point criterion.

\section{Criterion 3: Spectral resolution of the monochromatic optical monitoring system}

The spectral resolution of the monochromatic optical monitoring system is another important parameter to take into account when optimal optical monitoring strategy is being defined. Actually, due to interference effects in thin film filters, the spectral evolution of the transmittance of a filter can present broad and sharp spectral features. To illustrate this effect, we plotted in Figure 3 the spectral dependence of the transmittance, in the $450-850 \mathrm{~nm}$ spectral region, of a FabryPerot filter centered at $600 \mathrm{~nm}$ (blue curve). We then plotted the same transmittance curve when measured, not with an infinite spectral resolution, but with a finite spectral resolution of $1,2,3$ and $4 \mathrm{~nm}$. One can see that for such a filter, the spectral resolution can highly affect the local transmittance of the bandpass. Therefore, if this spectral resolution is not properly taken into account, the accuracy of the optical monitoring signal can be affected. This effect is a very wellknown effect [16] and is critical in this study.

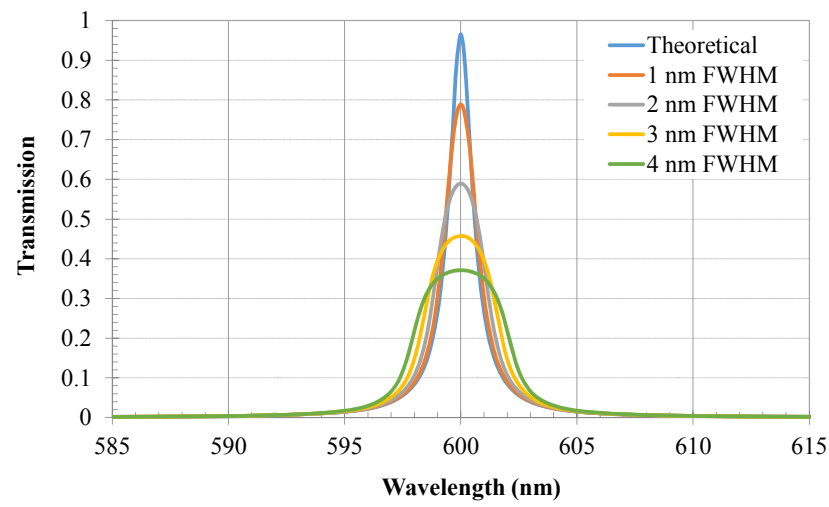

Figure 3. Illustration of a Fabry Perot filter measured with different resolutions from 1 to $4 \mathrm{~nm}$.

From this first result, it is obvious that if the spectral resolution of the optical monitoring system is finite and not compatible with the one of the filter, these wavelengths need to be removed from the potential optical monitoring wavelengths. To account for this effect, we defined a criterion that allows estimating the effect of the spectral resolution of the optical monitoring system on the measured transmittance. We calculated for each monitoring wavelength $\lambda_{n}$ and each layer $l_{n}$ a discrete quantity $E_{R}$ with unit $\% \mathrm{~T}$ and defined as:

$$
E_{R}\left(\lambda_{n}, l_{n}\right)=T\left(\lambda_{n}, l_{n}\right)-\frac{\sum_{\lambda_{i}=\lambda_{n}-R / 2}^{\lambda_{n}+R / 2} T\left(\lambda_{i}, l_{n}\right)}{N}
$$

with $\mathrm{R}$ being the full width spectral resolution in nanometer and $\mathrm{N}$, the number of points. This quantity quantifies the local effect, in percent, of the spectral resolution of the spectrometer for each monitoring wavelength and for each layer.

Using this criterion it is possible to generate two different regions:

- allowed regions where $\mathrm{E}_{\mathrm{R}}<1 \%$,

- forbidden regions where $\mathrm{E}_{\mathrm{R}}>1 \%$.

In the examples presented in this paper, we supposed a value of $\mathrm{R}$ of $2 \mathrm{~nm}$ and a step size of $0.1 \mathrm{~nm}$

\section{Criterion 4: Dynamic on every single layer}

The fourth criterion is related to the signal dynamic. Indeed, depending on the monitoring wavelength, the signal dynamic can be 
very limited. This is for example the case for the matching layer that are placed between two Fabry-Perot cavities in order to make then coherent. For these layers, when depositing a matching layer with a refractive index close to the one of the substrate, as the admittance of the stack is equal to the one of a bare substrate, the signal dynamic is close to zero at the central wavelength of the filter. In order to disregard the wavelengths that would exhibit such low signal dynamics, we defined a new criterion: transmittance change between the lowest and the highest measured transmittance of a layer must be larger than $5 \%$. Using this criterion it is possible to generate two different regions:

- allowed regions where transmittance change is larger than $5 \%$,

- forbidden regions where transmittance change is smaller than $5 \%$.

\section{E. Criterion 5: Transmittance value at the trigger point}

The fifth criterion is related to the transmittance level at trigger point. In order to secure as high as possible signal to noise ratio, one wants to avoid to have low transmission at trigger point. We therefore defined a criterion: transmittance must be larger than $10 \%$. Using this criterion it is possible to generate two different regions:

- allowed regions where transmittance larger than $10 \%$,

- forbidden regions where transmittance is smaller than $10 \%$.

\section{F. Criterion 6: Number of extrema during the deposition of a single layer}

The number of extrema that are crossed during the deposition of every single layers is also an important factor to take into account $[17,18]$. Indeed, even if it is not mandatory to cross some extrema for every single layer of a multilayer stack if the same wavelength is used throughout the whole deposition process, it is generally very beneficial as trigger point monitoring by swing (or POEM) algorithm recalculates trigger points based on the errors of amplitude of the measured signal at each extremum. In this case, partial phase compensation is performed. But such a criterion becomes of high interest as soon as multi-wavelengths optical monitoring is applied. When optical monitoring wavelength is changed, the error compensation as performed by trigger point monitoring by swing (or POEM) becomes not as easy to implement as signal modulation can vary a lot from one monitoring wavelength to another. If the signal of the first layer to be monitored with a new wavelength does not crosses an extremum, POEM will act as a simple trigger point monitoring technique and intensity errors associated with the errors on the previous layers will not be compensated. However, if the signal of the first layer to be monitored with a new wavelength crosses one extremum, and better two extrema, trigger point monitoring by swing (or POEM) procedure will allow an efficient compensation of the errors on previous layers by recalculating a new trigger point using the values of these extrema. Auto-compensation mechanism on the phase at the new monitored wavelength will be possible, and this correction will be effective for the following layers that will be monitored at the same wavelength.

In conclusion, it is particularly useful to change optical monitoring wavelength, every time it is possible, if one or two extrema are crossed in the monitoring of the first layer after this change. Using this criterion it is possible to generate two different regions:

- $\quad$ trigger point monitoring-optimized regions where the optical monitoring signal crosses two extrema,

- regular regions where the optical monitoring signal crosses zero or a single extremum.
Using this criterion, it is then straightforward to define regions where the change of optical monitoring wavelength would benefit from trigger point monitoring using swing (or POEM) error compensation.

\section{G. Description of the approach}

For each of these criteria, we have generated independent regions that are trigger point monitoring-compatible and other that are not. However, it is obvious that each of these criteria are important for trigger point monitoring such as it is important to take in account all of then simultaneously. To do so, we calculated a score where:

- Score of zero corresponds the case at least one of the five first criteria is not met. In this case optimal trigger point monitoring conditions are not combined for this layer and the associated monitoring wavelength should not be considered. These regions will be represented by grey rectangles.

- Score of one corresponds the case all five first criteria are met simultaneously. In this case optimal trigger point monitoring conditions are all combined for this layer and the associated monitoring wavelength can be considered. These regions will be represented by blue rectangles.

- Score of two corresponds the case all five first criteria are met simultaneously and the sixth criterion is also met. In this case optimal trigger point monitoring conditions are all combined for this layer and the associated monitoring wavelength can be considered. In addition, these regions are optimal regions for a change of the monitoring wavelength and will be represented by green rectangles.

Using these six criteria, it is then possible to generate trinary mappings for every new design that will provide an interesting visual input for the determination of an efficient optical monitoring strategy.

\section{Illustration of the approach on various examples}

\section{A. The quarter-wave mirror}

As a first example, we analyzed the trinary mapping technique on an 11-layer mirror centered at $600 \mathrm{~nm}$ composed with quarterwave layers at $600 \mathrm{~nm}$ with refractive indices of 2.35 and 1.46 deposited on a glass substrate with refractive index of 1.52 and with formula:

$$
\text { Glass }\left|(H L)^{5} H\right| \text { Air. }
$$

This example is particularly interesting to start with as classical Turning Point Monitoring at the central wavelength of a mirror is generally limited to low reflecting mirrors and finding an efficient trigger point monitoring wavelength is mandatory to produce high performance dielectric quarter wave mirrors. While this problem is not new, we provide here a comprehensive description on how to achieve an efficient optical monitoring strategy that will result in the lowest deviation from theory. In Figure 4, we plotted the evolution of trinary mapping of the mirror versus monitoring wavelength (horizontal axis) and layer number (vertical axis). These graphs read from bottom to top, i.e. from first to last layer of the stack and one looks for vertical paths that are all blue or green. One can see that as expected, $600 \mathrm{~nm}$ is not a wavelength that can be used for trigger point monitoring of a quarter-wave layer-based structure. Then, it appears that there are two windows that are trigger point monitoringcompatible that appear as blue or green continuous vertical lines. A first region on the short-wavelength edge of the mirror between 475 and $525 \mathrm{~nm}$ and a second region on the long-wavelength edge of the mirror between 710 and $765 \mathrm{~nm}$. With this technique, we have been able to eliminate $70 \%$ of the spectral regions that are not trigger point 
monitoring-compatible. The remaining regions corresponds to the regions on or close to the edges of the main reflection lobes of the mirrors and are in accordance with the well-known results for monitoring a quarter wave dielectric mirror.

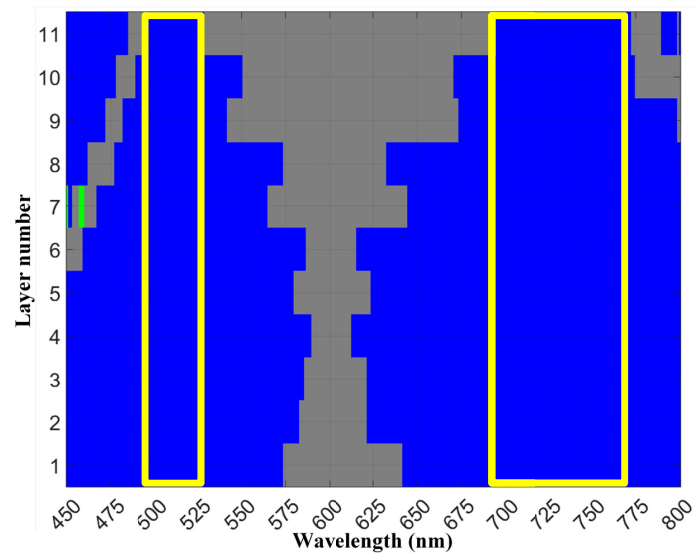

Figure 4 Trinary mapping of an 11-layer mirror stack in the [450 $800] \mathrm{nm}$ spectral region. Yellow rectangles show trigger point monitoring-compatible regions.

To select an appropriate strategy, one can either run virtual deposition process for different wavelengths within these ranges and select the one that results in the lowest discrepancy, or implement the technique that was recently presented [7] that consists in calculating the root means square deviation of the reflected phase at a single wavelength for each wavelength within the trigger point monitoringcompatible spectral regions and select the wavelength resulting in the lowest phase root mean square deviation.

By combining the trinary and phase method, it is then possible to generate, in an efficient way, an efficient optical monitoring strategy of an 11-layer mirror consisting in monitoring all the layers at a wavelength of $502 \mathrm{~nm}$.

\section{B. Narrow bandpass Fabry-Perot}

Another important filter example is a narrowband Fabry-Perot filter with formula M10 2H M10 centered at $600 \mathrm{~nm}$ with similar refractive indices as the one of the mirror with formula:

$$
\text { Glass }\left|(H L)^{5} 2 H(L H)^{5}\right| \text { Air. }
$$

Using the phase root mean square deviation at $600 \mathrm{~nm}$, we demonstrated that an efficient optical monitoring strategy consists in monitoring the 14 first layers using a trigger point monitoring at $497 \mathrm{~nm}$ and then switching to a turning point monitoring optical monitoring, when trigger point monitoring phase compensation becomes less efficient, in order to guaranty perfect centering of the filter [7]. However, determining the optimal trigger point monitoring wavelength is very time consuming as it requires calculating this phase parameters for each possible monitoring wavelength. We therefore investigated if trinary mappings can be used to restrict the range of possible spectral ranges where phase root mean square deviation would be calculated. Trinary mapping of the considered Fabry-Perot filter was generated (Figure 5). One can see again that, as expected, $600 \mathrm{~nm}$ is not a wavelength that can be used for P trigger point monitoring of a quarter-wave layers-based structure. Then, it appears that there are four narrow windows that are trigger point monitoringcompatible for the first 14 layers and that appear as blue or green continuous vertical lines. Two first regions on the short-wavelength edge of the mirror between 475 and $500 \mathrm{~nm}$ and between 505 and
$512 \mathrm{~nm}$ and two additional regions on the long-wavelength edge of the mirror between 720 and $740 \mathrm{~nm}$ and between 762 and $782 \mathrm{~nm}$. Similarly to the mirror, using the trinary mappings, we have been able to eliminate $80 \%$ of the wavelengths that are not trigger point monitoring-compatible.

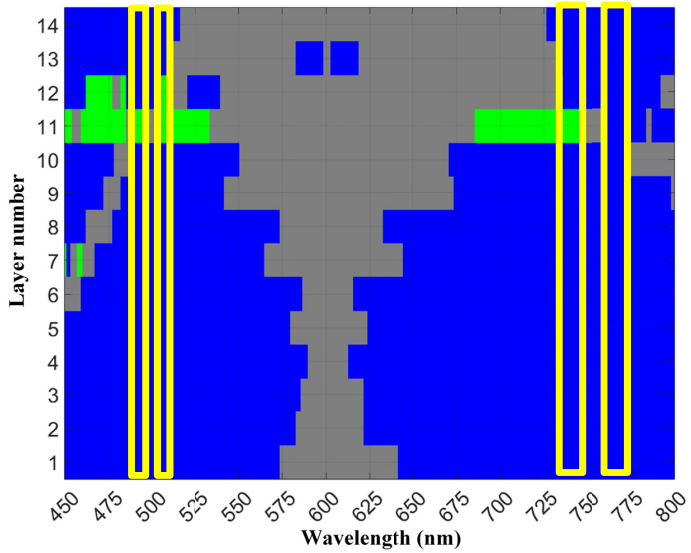

Figure 5 Trinary mapping of the first 14 Fabry-Perot layers [450 $800] \mathrm{nm}$. Yellow rectangles show trigger point monitoring-compatible regions.

We then overlapped these regions with the evolution of the phase root mean square deviation ( $\varphi_{\mathrm{RMSD}}$ ) of the $14^{\text {th }}$ layer of the mirror as calculated in ref [6] (Figure 6). The grey regions correspond to the regions that are not trigger point monitoring-compatible and the blue regions the regions that are trigger point monitoring-compatible for the $14^{\text {th }}$ first layers.

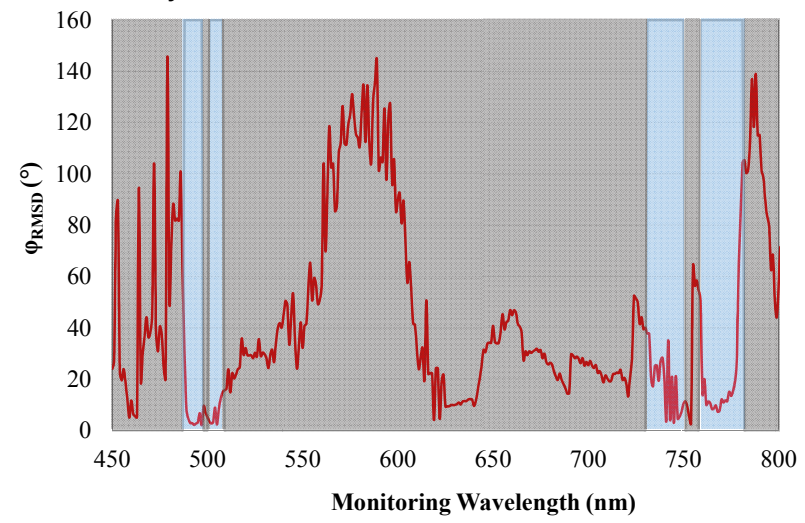

Figure 6 Comparison of the final layer phase root means square deviation $\left(\varphi_{\mathrm{RMSD}}\right)$ mapping and the trigger point monitoringcompatible spectral regions as selected by the trinary mapping of the first 14 Fabry-Perot layers.

One can see that the regions selected by the trinary mapping perfectly coincide with the regions where the phase root mean square deviation is minimum. In other words, we see that while the trinary mappings cannot provide an efficient optical monitoring strategy, they can be used as a method to limit the range of optical monitoring wavelengths that are scanned for the calculation of the root mean square deviation, severely reducing the number of calculations. By combining these two methods, it is then possible to generate, in an efficient way, an efficient optical monitoring strategy of the $14^{\text {th }}$ first layers of a M10 2H M10 Fabry-Perot filter, i.e. the monitoring of all layers at a wavelength of $497 \mathrm{~nm}$. 
One can also see that while the trinary mapping could select the regions associated with the lowest phase root mean square deviation, it did not select a region in the $625-650 \mathrm{~nm}$ where the phase root mean square deviation appears also pretty small. The reason for not selecting this zone is associated with the low intensity modulation, especially in and close to the spacer. From a practical point of view, the combination of the trinary mapping and phase root mean square deviation is a simple and pretty fast technique for determining, if not the optimal, an efficient all-optical monitoring technique for any thin film optical component.

\section{Non-conventional three cavity bandpass filter}

In ref [19], a multi-cavity Fabry-Perot filter based on non-quarter wave layers and centered at $600 \mathrm{~nm}$ proposed by R. Willey was studied. The stack formula is given below:

Glass | $(0.5 H 1.5 \mathrm{~L})^{3} 3.632 \mathrm{H}(1.5 \mathrm{~L} 0.5 \mathrm{H})^{3} 1.345 \mathrm{~L}$

$(0.5 H 1.5 L)^{3} 3.632 H(1.5 L 0.5 H)^{3} 1.345 L(0.5 H 1.5 L)^{3}$

3.632H $(1.5 \mathrm{~L} 0.5 H)^{3} 1.19989 \mathrm{~L} 0.96265 H 0.90522 \mathrm{~L} \mid$ Air.

Using phase root mean square deviation, it was demonstrated that there is a better optical monitoring strategy than monitoring the stack at $600 \mathrm{~nm}$ [6]. This efficient optical monitoring strategy consists in monitoring the 37 first layers using a trigger point monitoring at $596 \mathrm{~nm}$ and then monitoring the 5 last layers using a trigger point monitoring at $606 \mathrm{~nm}$. The determination of this strategy required once more to determine for each wavelength and each layer the phase root mean square deviation which is once more time consuming. In this paper, we implemented the trinary mapping technique for this filter (Figure 7). One can see that $600 \mathrm{~nm}$ is not a wavelength that can be used for trigger point monitoring of all the layers. To overcome this problem, R. Willey proposed to quartz monitor layers 14, 27, 28, 40, 41 and 42 . We see on the trinary mapping that based on the limits that we have applied for each criterion, these layers are not trigger point monitoring-compatible and should effectively be quartz monitored.

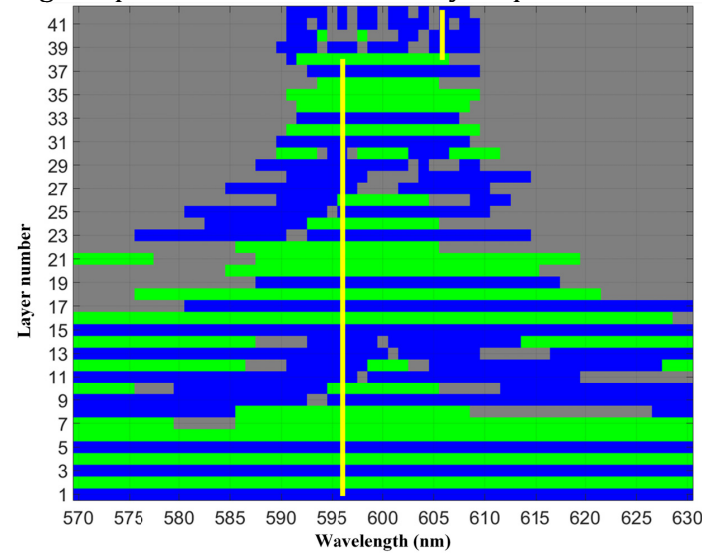

Figure 7 Trinary mapping of a non-conventional Fabry-Perot filter [570 - 630] nm. Optimal path is highlighted with yellow lines.

Then, if one looks at the overall trinary mapping, it appears that there is no complete blue/green vertical path, i.e. there is no single wavelength that allows using trigger point monitoring for the whole stack. However, $596 \mathrm{~nm}$ appears as the wavelength that allows monitoring the largest number of layer with a single wavelength 1 to 40 (blue or green continuous vertical lines highlighted with a yellow line). In this case, trinary mapping did not result in a spectral region of interest but in a single optimal optical monitoring wavelength: $596 \mathrm{~nm}$.

One can see that trinary mapping provides similar information as the one that was obtained from phase root mean square deviation. However, while the trinary mapping showed that $596 \mathrm{~nm}$ is trigger point monitoring-compatible until layer 39 , phase root mean square deviation showed that phase error increases too much for the last 2 layers and a change of monitoring wavelength at layer 38 would allow minimizing manufacturing errors. This change of monitoring wavelength at layer 38 also appears as optimal in the trinary mapping as it corresponds to a green region, i.e. corresponding to regions with an optical monitoring signal that crosses two extrema before reaching the trigger point.

For the monitoring of the last 5 layers, one can see on the trinary mapping that there are two wavelengths that are trigger point monitoring-compatible: 603 and $606 \mathrm{~nm}$. One can see that once more, trinary mapping includes the wavelength that was determined from phase root mean square deviation and is therefore an efficient way to restrict the number of possible optical monitoring strategies.

\section{Antireflection coating}

The last standard thin film optical component that was studied is a broadband antireflection (AR) coating covering the $550-650 \mathrm{~nm}$ region with formula:

Glass | $0.1703 \mathrm{H} \quad 0.4459 \mathrm{~L} \quad 2.0544 \mathrm{H} \quad 1.5162 \mathrm{~L} \quad 0.2354 \mathrm{H}$

$0.2485 \mathrm{~L} 1.1641 \mathrm{H} 0.9651 \mathrm{~L} / \mathrm{Air}$.

Antireflection coating is hard to monitor due to its low reflective nature. We restricted this analysis to the first 7 layers. Indeed, in order to achieve perfect AR coatings, the admittance of the stack after the $8^{\text {th }}$ layer must be equal to the one of the incident medium, i.e. air for each wavelength between 550 and $650 \mathrm{~nm}$. In other words, monitoring the transmittance of the last layer is not trigger point monitoringcompatible and the triggering method is then limited to turning point monitoring or quartz monitoring. We plotted in Figure 8 the trinary mapping of the AR coating. One can see that there is a single region from 460 to $500 \mathrm{~nm}$ that is compatible with single wavelength optical monitoring based on the criteria that we have defined. Main limitation is on layers 2 and 6 that are not trigger point monitoring-compatible. We plotted in Figure 9 an overlap between the trinary mapping and phase root mean square deviation of the last layer. One can also see that there is a good correlation between these two criteria. Regions associated with a trigger point monitoring-incompatible layer result in higher phase errors the larger the number of trigger point monitoringincompatible layers, the higher the phase error. We clearly see that by combining these two techniques, one can quickly converge to an efficient optical monitoring procedure.

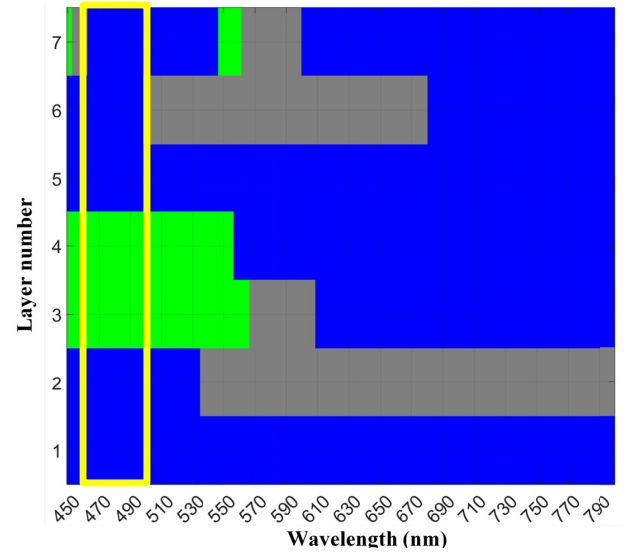

Figure 8 Trinary mapping of the 7 first layers on an antireflection coating. Yellow rectangle shows trigger point monitoring-compatible region. 


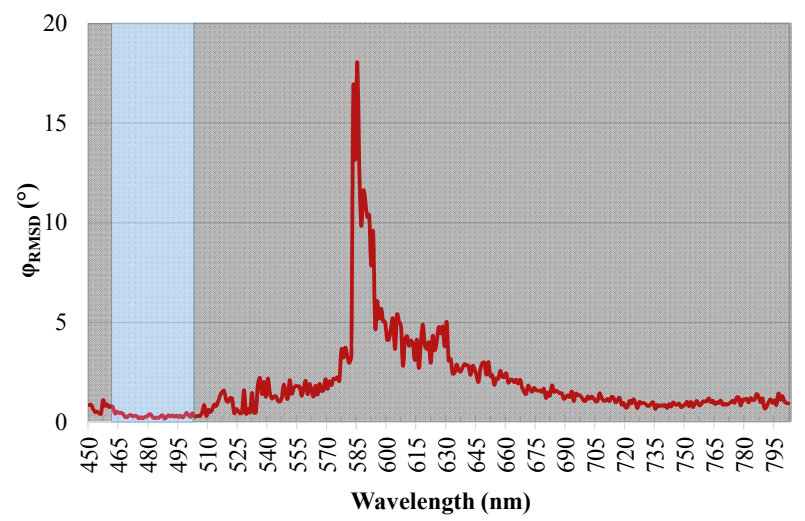

Figure 9 Comparison of the final layer phase root mean square deviation ( $\varphi$ RMSD) at 7th layer and the trigger point monitoringcompatible spectral regions as defined by trinary mappings.

However, we see that these two techniques did not permit to determine one single strategy but more a spectral range to select a monitoring wavelength. One can show that almost whatever the wavelength used in this range, good theory/experiment will be achieved. However, by running virtual deposition process for all the wavelength in this range, the strategy consisting in monitoring layers [1-8] at $488 \mathrm{~nm}$ can be shown to result in the lowest phase root mean square deviation and the spectral performances the closest to the theoretical one.

\section{Experimental validations}

We experimentally validated the determined strategies by depositing the thin film components using a Plasma Assisted Reactive Magnetron Sputtering (PARMS, Bühler HELIOS machine) combined with a Bühler OMS 5000 optical monitoring system. Each of the studied components were fabricated, except for the Fabry-Perot filter as the OMS 5000 did not allow easily switching from trigger point monitoring to turning point monitoring. For each of the components, the strategy was slightly adapted in order to account for refractive index dispersion of the HELIOS machine $\left(\mathrm{Nb}_{2} \mathrm{O}_{5}\right.$ and $\left.\mathrm{SiO}_{2}\right)$, but the determined wavelength did not deviate by more than a few nanometers compared to the one determined in section 3 without dispersion as the considered refractive indices are close to the one of the HELIOS machine. Finally, all samples were characterized using a Perkin Elmer Lambda 1050 spectrophotometer. We plotted in Figure 10 an overlay between theoretical and experimental performances.

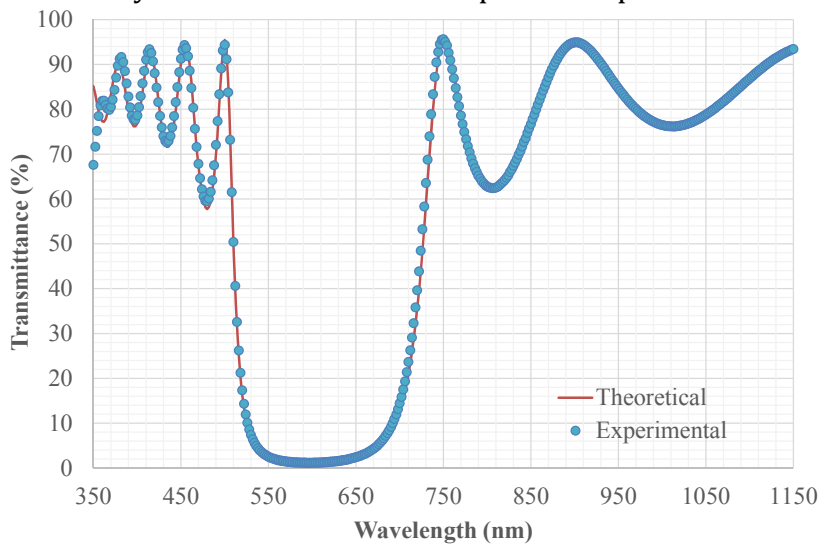

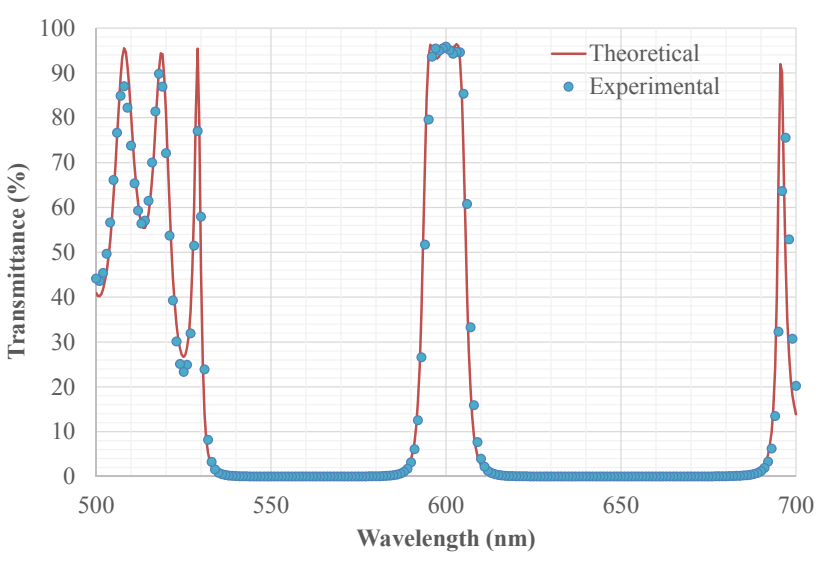

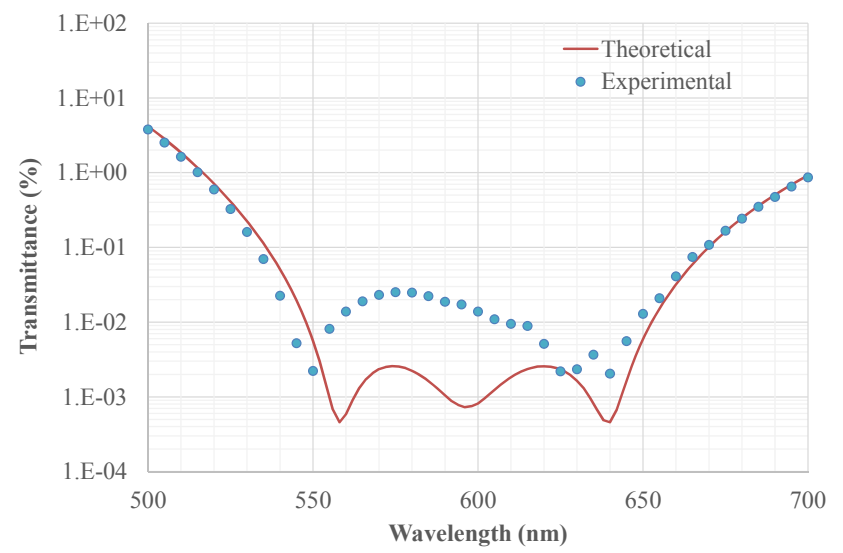

Figure 10 Comparison the theoretical and experimental transmission/reflection in case of a) a mirror, b) a multicavity bandpass filter and c) an antireflection coating.

Regarding the mirror, one can see that trigger point monitoringmonitored mirror shows almost no measurable deviation from the theoretical profile, confirming that selecting a wavelength on the shortwavelength side of the main reflection lobe is an efficient strategy.

Regarding the multi-cavity bandpass filter, one can see a good theoretical/experimental agreement both close to the bandpass and over a broader spectral range. The central wavelength is shifted by $\sim 0.5 \mathrm{~nm}$ and the main discrepancies in broadband domain are associated with the limited resolution of the spectrophotometer (here $1 \mathrm{~nm}$ ) to maximize the signal-to-noise ratio. This result confirms that while Willey proposed to optically monitor most of the filter at $600 \mathrm{~nm}$ (except for some layers that need to be quartz-crystal monitored), a slight adjustment by a few nanometers of the monitoring wavelength allows accurate all-optical monitoring of this kind of filter.

Finally, one can see that we have been able to fabricate an antireflection coating with residual reflection within the [550-650] nm spectral range not exceeding $0.02 \%$, confirming that the all-optical strategy is valid and efficient. Over a broad spectral range, the two curves match very well proving that we are very close to theoretical performances. In the low reflection zone, one can see that the discrepancy is lower than one order of magnitude. It can be due to limited sensitivity and precision of the spectrophotometer or very small errors that should not exceed $0.2 \mathrm{~nm}$ for each layer. In conclusion, this experimental demonstration shows that the proposed strategy is also compatible with all-optical monitoring of this type of optical function. 


\section{Conclusions}

We have developed a new approach for the determination of efficient all-optical monitoring strategy for the manufacturing of thin film optical components. It is based on the generation of a trinary mappings that allow defining a range of possible wavelengths that are trigger point monitoring-compatible. These mappings that do not rely on virtual deposition process, but on an analysis of the optical monitoring signals obtained for all monitoring wavelengths, using some criteria and applying some limits to determine whether a wavelength can be used for the optical monitoring of its thickness or not. This method does not provide the best optical monitoring strategy, but a range of possible one. This technique can then be combined with a technique based on phase root mean square deviation analysis at a single representative wavelength ( $\varphi$ RMSD). The wavelength that allows minimizing $\varphi_{\mathrm{RMSD}}$ is then selected an optimal optical monitoring wavelength. By combining these two techniques, it is then possible to find an efficient optical monitoring strategy. This approach was illustrated on different examples including mirror, bandpass filters and antireflection coatings. For each of them we determined a single all optical monitoring strategy that was validated using virtual depositions process. It is finally worth noting that all these results were experimentally validated by implementing these strategies on plasma Assisted Reactive Magnetron Sputtering machines.

Acknowledgment. This work was performed within the LabTOP (Laboratoire commun de Traitement OPtique des surfaces), a research cooperation agreement between Institut Fresnel (Aix-Marseille Univ, CNRS, Centrale Marseille) and CILAS Company.

\section{References}

1. K. D. Hendrix; C. A. Hulse; G. J. Ockenfuss; R. B. Sargent, "Demonstration of narrowband notch and multi-notch filters," Proc. SPIE 7067, Advances in Thin-Film Coatings for Optical Applications V, 706702 (29 August 2008).

2. M. Scherer, J. Pistner, and W. Lehnert, "UV- and VIS filter coatings by plasma assisted reactive magnetron sputtering (PARMS)," in Optical Interference Coatings, OSA Technical Digest (Optical Society of America, 2010), paper MA7.

3. T. Begou, H. Krol, D. Stojcevski, F. Lemarchand, M. Lequime, C. GrezesBesset and J. Lumeau, "Complex optical interference filters with stress compensation for space applications", CEAS Space J 9(4), 441-449 (2017).

4. R. R. Willey, "Simulation comparisons of monitoring strategies in narrow bandpass filters and antireflection coatings," Appl. Opt. 53, A27-A34 (2014).

5. H. A. Macleod, Thin-Film Optical Filters, 4th ed., (CRC Press/Taylor \& Francis, 2010).

6. M. Vignaux, F. Lemarchand, T. Begou, C. Grezes-Besset and J. Lumeau, "In-situ optical monitoring of Fabry-Perot multilayer structures: analysis of current techniques and optimized procedures", Optics Express 25, 18040-18055 (2017).

7. T. Begou, F. Lemarchand and J. Lumeau, "Advanced optical interference filters based on metal and dielectric layers", Optics Express 24(18), 20925-20937 (2016).

8. R. R. Willey, "Design and monitoring of narrow bandpass filters composed of non-quarter-wave thicknesses," SPIE vol. p. 7101-19 (2008).

9. R. R. Willey "Preserving error compensation benefits while changing monitoring wavelengths with each layer," Willey Optical, http://www.willeyoptical.com/.

10. R. Willey and A. Zöller, "Computer simulation of monitoring of narrow bandpass filters at nonturning points," Society of Vacuum Coaters Annual Technical Conference Proceedings 52, $432-437$ (2009).
11. A. Zöller, M. Boos, R. Götzelmann, H. Hagedorn, B. Romanov, and M. Viet, "Accuracy and error compensation with direct monochromatic monitoring," in Optical Interference Coatings, OSA Technical Digest (Optical Society of America, 2013), paper WB5.

12. A. Zöller, M. Boos, H. Hagedorn, B. Romanov, "Computer simulation of coating processes with monochromatic monitoring," SPIE 7101-0G (2008).

13. M. Trubetskov, T. Amotchkina, and A. Tikhonravov, "Automated construction of monochromatic monitoring strategies," Appl. Opt. 54, 1900-1909 (2015).

14. J. A. Dobrowolski, "Modern computational methods for optical thin film systems," Thin Solid Films 34, 313-321 (1976).

15. A. V. Tikhonravov, M. K. Trubetskov, "OptiLayer software," http://www.optilayer.com.

16. A. Zoeller, M. Boos, R. Herrmann, W. Klug, W. Lehnert, "Optical thickness monitoring of dielectric optical filters using a new in-situ photometer with high signal resolution and excellent long term stability," SPIE 1019-14, (1988).

17. R. R. Willey "Preserving Error Compensation Benefits While Changing Monitoring Wavelengths With Each Layer," Willey Optical, http://www.willeyoptical.com/.

18. R. R. Willey, "Non-turning-point monitoring improves narrow bandpass filters," Appl. Opt. 48, 3277-3283 (2009).

19. R. R. Willey, "Practical Design and Production of Optical Thin Films," CRC press (2002). 